\title{
Low energy radioactivity BG model in Super-Kamiokande detector from SK-IV data
}

\section{Guillaume Pronost ${ }^{a, *}$ on behalf of the Super-Kamiokande Collaboration}

(a complete list of authors can be found at the end of the proceedings)

\author{
${ }^{a}$ Kamioka Observatory, ICRR, The University of Tokyo, \\ Kamioka, Gifu 506-1205, Japan \\ E-mail: pronost@km.icrr.u-tokyo.ac.jp
}

The radioactivity background are among the most dangerous background for low energy neutrino analysis in Super-Kamiokande (SK), like the solar neutrino analysis. Among them, the main contribution is coming from ${ }^{222} \mathrm{Rn}$, which is spread in the detector's water due to the water source and to the photo multiplier (PMT) emanations. Up to now, its exact distribution in the detector was not known. Using our knowledge of the radon concentration in the detector water, and the SK-IV solar data, we developed a model of the radon distribution in the detector. The uncertainty on the Rn concentration associated with this model was estimated to be $\sim 0.1 \mathrm{mBq} / \mathrm{m}^{3}$

$37^{\text {th }}$ International Cosmic Ray Conference (ICRC 2021)

July 12th-23rd, 2021

Online - Berlin, Germany

\footnotetext{
${ }^{*}$ Presenter
} 


\section{Introduction}

The Super-Kamiokande (Super-K) experiment[1] is a successful neutrino experiment located in the Kamioka mine, about $1 \mathrm{~km}$ below the top of Ikenoyama, in Kamioka-cho (Japan). Super-K uses a 50 ktons water Čerenkov detector, operating since 1996. The experiment is dedicated to neutrino and proton-decay researches, among them the low energy solar neutrino analysis suffer from the presence of ${ }^{222} \mathrm{Rn}$ (thereafter noted as "Rn"), a radioactive gas dissolved in water, and a major source of backgrounds (BGs) from this analysis.

The impact of Rn on the low energy analysis comes from the presence of ${ }^{214} \mathrm{Bi}$ in its decaychain. ${ }^{214} \mathrm{Bi}$ is a $\beta$ emitter, with a $\mathrm{Q}$ value of $3.2 \mathrm{MeV}$. In Super-Kamiokande, these radioactive decays are the dominant $\mathrm{BG}$ below $5 \mathrm{MeV}$. In order to reduce the Rn concentration in the water, Super-K used some dedicated methods and device in its water purification system [2], allowing to reduce the input water's Rn concentration, as well as to generate a stagnated water region in the center of the detector, where Rn decays without being replaced, producing a low Rn concentration region in the center of the detector.

Despite these efforts, Rn can not be completely removed from the Super-K's water, and is still affecting the solar neutrino analysis. In order to understand its impact on the analysis, the Super-K collaborators conducts periodic direct measurement of the Rn concentration [2], as well as Rn-rich water injection [3], in the detector water. These measurements provide an idea of the Rn BG rate in the analysis, as function of the Rn concentration.

However, these direct Rn measurements are restricted to a few fixed position in the detector from where the water can be sampled. Other methods are needed in order to understand the distribution of $\mathrm{Rn}$ in the full detector. In this study, we compared the event rate in Super-K data with MC simulations in order to estimate the Rn concentration in each point of the detector. This approach was inspired by [3], where the event rate is compared with the Rn concentration in the injected water.

\section{Super-Kamiokande data and simulation}

\subsection{Data selection}

For this study, we needed to use a solar neutrino analysis data sample with a large statistics, stable Rn concentration in the detector (i.e. stable water system configuration), and with stable detection efficiency. We selected the period from May 2015 to February 2018, during which the SLE (Super Low Energy) trigger threshold was minimum. In this data sample, we applied the same selection criteria than for the solar neutrino analysis [4], the angle between the event's reconstructed directions and the sun was not considered. In order to select the energy region affected by the Rn BG, we only selected events with a reconstructed kinetic energy from 3.5 to $4.5 \mathrm{MeV}$. For this selected period, the data sample lifetime is 836 days. Fig. 1 shows the $2 \mathrm{D}$ vertex distribution of the selected events.

The solar neutrino event rate in this energy range is expected to be $\sim 0.5 \times 10^{-3}$ event $/ \mathrm{day} / \mathrm{m}^{3}$. Therefore, we assumed all events in the selected data sample are due to the Rn BG. 


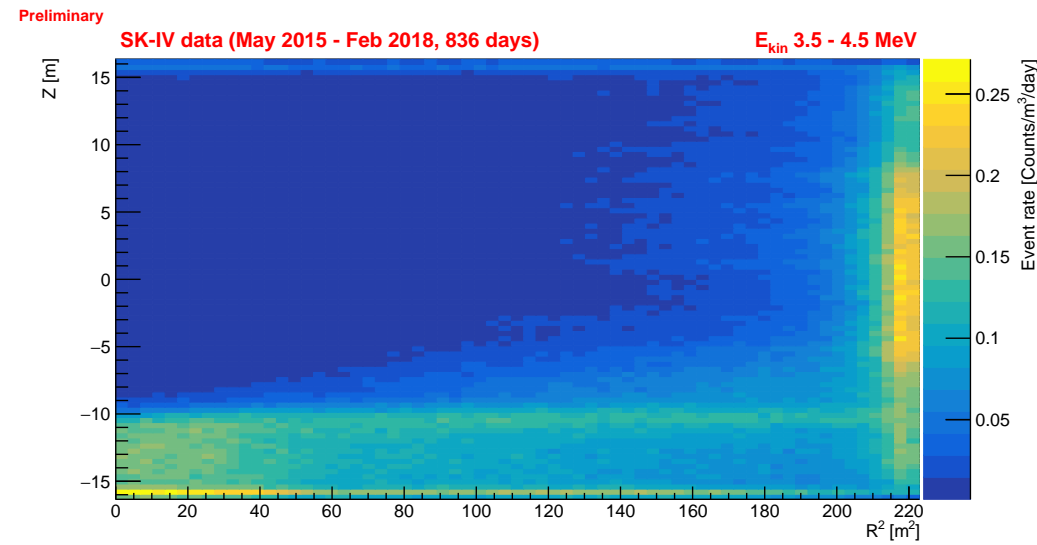

Figure 1: $2 \mathrm{D}$ distribution of the event rate in Super-K solar neutrino analysis for $\mathrm{E}_{k i n} \in[3.5,4.5] \mathrm{MeV}$

\subsection{Simulation}

In this study, we assumed the Rn related BG is only due to ${ }^{214} \mathrm{Bi} \beta$ decays, and thus we generated a sample consisting only in ${ }^{214} \mathrm{Bi} \beta$ decays. The ${ }^{214} \mathrm{Bi}$ decay were generated with a Geant4-based MC simulation[5-7] using the default RadioactiveDecay and PhotonEvaporation models for geant4-10.03p3. Particles produced from the decays (i.e. $\mathrm{e}^{-}$and deexcitation $\gamma \mathrm{s}$ ) were injected in the Super-K Geant4-based MC simulation, skdetsim. These ${ }^{214} \mathrm{Bi}$ decays were generated uniformly in the full Super-K's inner detector. We applied the same selection criteria as for data on the simulation output.

Multiple large statistics $\left(O\left(10^{9}\right)\right.$ events) samples were produced, in order to build and validate the model with independent samples.

\section{Model construction}

As shown in Fig. 1, the event distribution in the detector is not uniform, and complex structure can be observed, as around $\mathrm{Z}=-10 \mathrm{~m}$, or in the bottom corner of the detector. In order to build a model of the Rn concentration, these structures need to be taken into account. As working with 2D-distributions is impracticable, we divided the detector volume in different layers following the $\mathrm{Z}$ - $\mathrm{R}^{2}$-axis, for each layer we obtained a $1 \mathrm{D}$-event rate distributions.

We divided the detector into sixteen $17.75 \mathrm{~m}^{2}$-width layers following the $\mathrm{R}^{2}$-axis, and eighteen $2 \mathrm{~m}$-width layers following the $\mathrm{Z}$-axis. The number of layer was defined in order to keep high enough statistics and to avoid averaging the complex structure observed in the data. Some layers are actually outside the fiducial volume used by the solar neutrino analysis, hence they were not used for this study. Fig. 2 and 3 show an example of the event rate distribution in one Z-layer and one $\mathrm{R}^{2}$-layer, respectively.

\section{1 ${ }^{208}$ Tl decays}

In Fig. 2, an exponential increase is visible at the very borders of the fiducial volume, below $-15 \mathrm{~m}$ and above $15 \mathrm{~m}$. Similar increase are also present in the $\mathrm{R}^{2}$-layers. These event rate increases were identified using MC simulation as compatible with another BG source in the detector: ${ }^{208} \mathrm{Tl}$. 


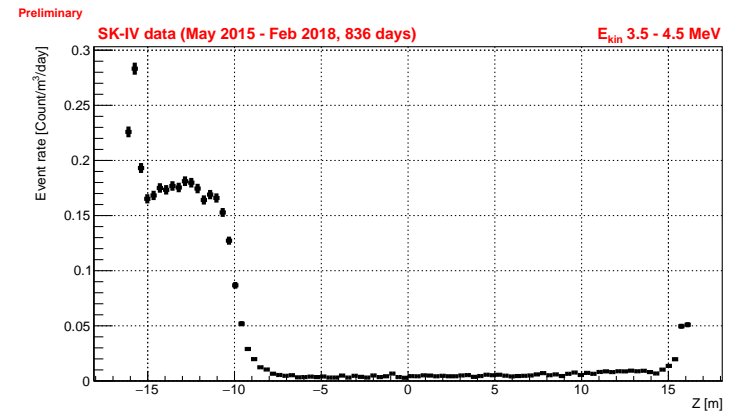

Figure 2: Event rate in data for the Z-layer selected with $\mathrm{R}^{2}<17.75 \mathrm{~m}^{2}$

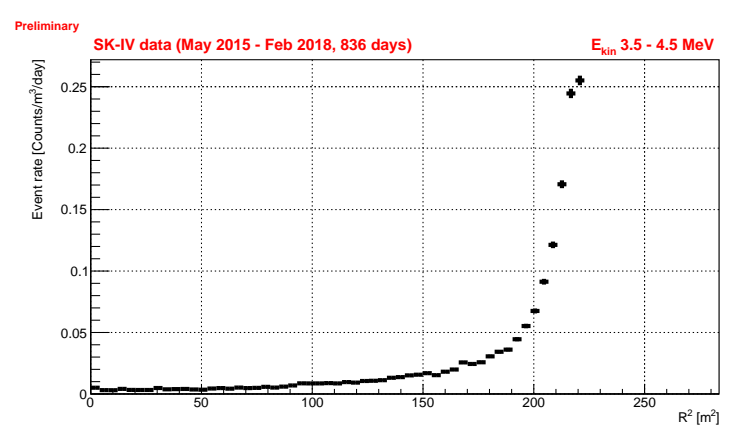

Figure 3: Event rate in data for the $\mathrm{R}^{2}$-layer selected with $\mathrm{Z} \in[-0.8,0.8] \mathrm{m}$

${ }^{208} \mathrm{Tl}$ decays with the production of a $2.6 \mathrm{MeV} \gamma$, producing $\mathrm{BG}$ events in the same energy range as Rn. In the Super-K detector, we identified two large sources of ${ }^{208} \mathrm{Tl}$ : the photo multiplier (PMT) glass, as well as the PMT resin-covers. Taken into account together, the ${ }^{208} \mathrm{Tl}$ decay rate is estimated to be $\sim 42 \mathrm{~Bq} / \mathrm{PMT}$ ( $\sim 14 \mathrm{~Bq} / \mathrm{PMT}$ for the top PMTs). ${ }^{208} \mathrm{Tl}$ sources being located at the border of the detector, most of the ${ }^{208} \mathrm{Tl}$ events are rejected by the selection cuts, but a non-negligible amount is selected in our data sample.

As a temporary solution for the model construction, in order to exclude the ${ }^{208} \mathrm{Tl}$ decays, we constrained the analysis volume to $\mathrm{Z} \in[-14.8,14.8] \mathrm{m}$ and $\mathrm{R}^{2}<200 \mathrm{~m}^{2}$.

\subsection{Model construction procedure}

The construction of the Rn distribution model was made in three steps: the Rn concentration extraction from the data for each $\mathrm{Z}$ - and $\mathrm{R}^{2}$-layers; the fit of the $\mathrm{Rn}$ concentration distributions; and the interpolation of the Rn concentration fit to build a 2D-model.

\subsubsection{Rn concentration extraction}

In order to extract the Rn concentration for the data, we arbitrary scaled the Rn MC sample to $1 \mathrm{~Bq} / \mathrm{m}^{3}$ by applying a scaling factor, $1 / f$ (bin) on each bin of the distribution. $f$ (bin) was defined as follow:

$$
f(\text { bin })=\frac{\# \text { Simulated decays }}{1 \mathrm{mBq} / \mathrm{m}^{3} \times \text { Volume }(\mathrm{ID}) \times 86400 \mathrm{sec} / \text { day }} \times \text { Volume }(\text { bin })
$$

The Rn concentration $C$, in $\mathrm{Bq} / \mathrm{m}^{3}$, was extracted for each bin as follow:

$$
C(\text { bin })=\frac{\text { rate }^{\mathrm{data}}(\text { bin })}{\text { rate }^{\mathrm{MC}}(\text { bin })}
$$

Fig. 4 and 5 show an example of the extracted Rn concentration in one Z-layer and one $\mathrm{R}^{2}$-layer, respectively.

\subsubsection{Rn concentration fit}

In order to get a linear expression of the Rn concentration in each layer, we fitted each extracted $\mathrm{Rn}$ concentration distributions with a function representing the $\mathrm{Rn}$ distribution in a layer. This fit function was build considering two simple cases for the Rn distribution in the detector: 
- Regions of the detector in which the Rn concentration is uniform

- Regions of the detector in which the $\mathrm{Rn}$ is diffusing from one position to another

We modeled the first case with constant functions, and the second case with inverse hyperbolic cosine function[8] defined as:

$$
D(x, \mathrm{X}, \mathrm{S})=\frac{1}{\cosh \left((x-\mathrm{X}) \times \lambda_{\mathrm{Rn}} / \mathrm{S}\right)}
$$

with $x$ the current position, $\mathrm{X}$ the position of the diffusion origin, and $\mathrm{S}$ the diffusion factor. $\lambda_{\mathrm{Rn}}$ is the Rn decay constant.

The fit function applied on the Z-layers was defined as:

$$
f_{Z}(x)= \begin{cases}\mathrm{C} 2+(\mathrm{C} 1-\mathrm{C} 2) * D(x, \mathrm{X} 2, \mathrm{~S} 2), & \text { if } x<\mathrm{X} 2 \\ \mathrm{C} 1, & \text { if } x<\mathrm{X} 1 \text { and } x>\mathrm{X} 2 \\ \mathrm{C} 0+(\mathrm{C} 1-\mathrm{C} 0) * D(x, \mathrm{X} 1, \mathrm{~S} 1), & \text { otherwise }\end{cases}
$$

with $\mathrm{C} 0, \mathrm{C} 1, \mathrm{C} 2, \mathrm{X} 1, \mathrm{X} 2, \mathrm{~S} 1$, and $\mathrm{S} 2$, the fit parameters. The fit function applied on the $\mathrm{R}^{2}$-layers was defined as:

$$
f_{R^{2}}(x)= \begin{cases}\mathrm{C} 2, & \text { if } x>\mathrm{X} 2 \\ \mathrm{C} 1+(\mathrm{C} 2-\mathrm{C} 1) * D(\sqrt{x}, \sqrt{\mathrm{X} 2}, \mathrm{~S} 2), & \text { if } x>\mathrm{X} 1 \text { and } x<\mathrm{X} 2 \\ \mathrm{C} 0+(\mathrm{C} 2-\mathrm{C} 1) * D(\sqrt{x}, \sqrt{\mathrm{X} 2}, \mathrm{~S} 2) & \\ \quad+(\mathrm{C} 1-\mathrm{C} 0) * D(\sqrt{x}, \sqrt{\mathrm{X} 1}, \mathrm{~S} 1), & \text { otherwise }\end{cases}
$$

with $\mathrm{C} 0, \mathrm{C} 1, \mathrm{C} 2, \mathrm{X} 1, \mathrm{X} 2, \mathrm{~S} 1$, and $\mathrm{S} 2$, the fit parameters.

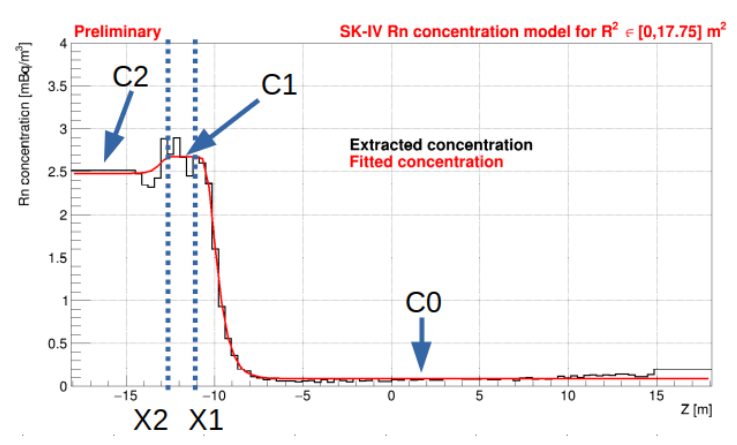

Figure 4: Extracted Rn distribution for Z-layer (black) selected with $\mathrm{R}^{2}<17.75 \mathrm{~m}^{2}$ and fitted by Eq. 4 (red). C0, C1, C2, X1, and X2 illustrate the fit parameter values for this particular layer.

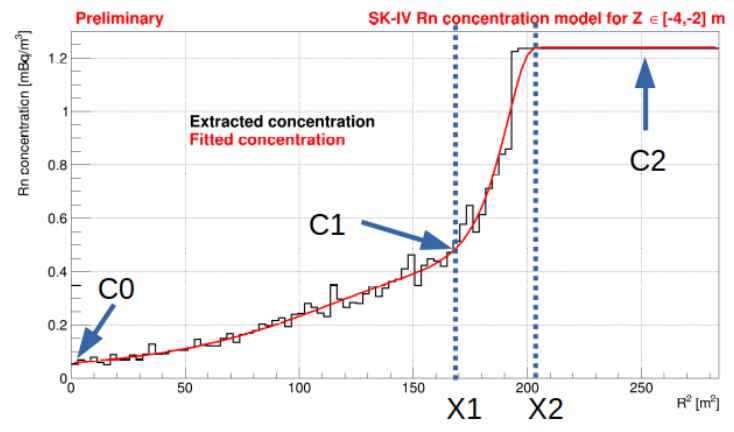

Figure 5: Extracted $\mathrm{Rn}$ distribution for $\mathrm{R}^{2}$-layer (black) selected with $\mathrm{Z} \in[-4,-2] \mathrm{m}$ and fitted by Eq. 5 (red). C0, C1, C2, X1, and X2 illustrate the fit parameter values for this particular layer.

\subsubsection{D-model construction}

The Rn distribution model is constructed by interpolation between the $10 \mathrm{Rn}$ concentration functions for $\mathrm{R}^{2}$-layers and the $12 \mathrm{Rn}$ concentration functions for Z-layers. For each position, the 
$\mathrm{Rn}$ concentration is calculated separately using the Z-layer functions and the $\mathrm{R}^{2}$-layer functions. The minimum concentration between both is then selected as the final Rn concentration. Model made with the average concentration, or with the maximum concentration, were also tested but show a poorer agreement with data.

However, in the detector borders, the interpolation is made between the closest layer function and the expected Rn concentration due to PMT material's Rn emanation, in this case, the maximum concentration is used instead of the minimum.

Fig. 6 shows the resulted model of the Rn distribution in the Super-K detector.

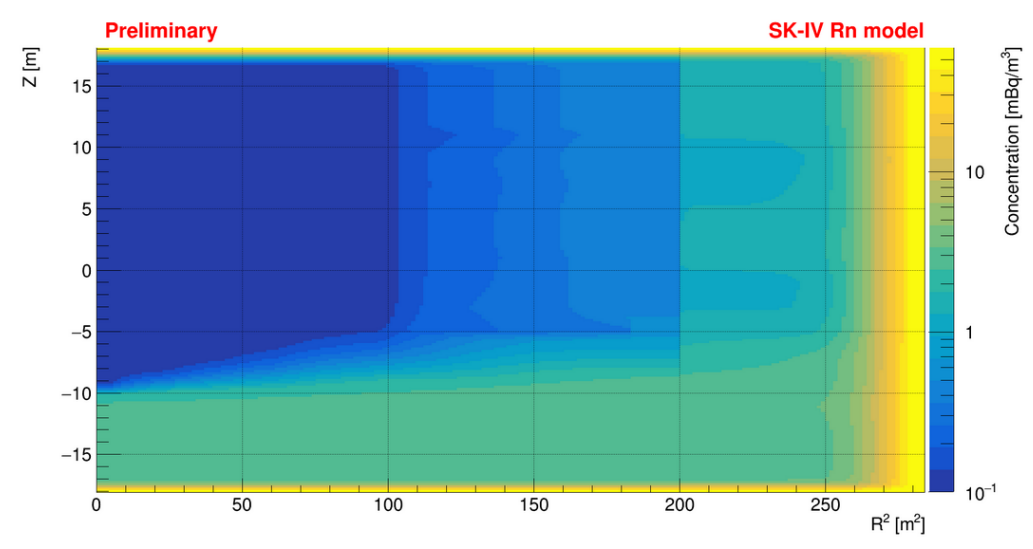

Figure 6: 2D-model of the Rn distribution in the detector

\section{Model validation}

In order to validate the model presented in Fig. 6, we generate a new ${ }^{214} \mathrm{Bi}$ decays $\mathrm{MC}$ sample and scaled it following the Rn model. The uncertainty on the Rn model was then calculated as follow:

$$
\operatorname{err}_{\mathrm{Rn}}(\text { bin })=\left(1-\frac{\operatorname{rate}^{\mathrm{data}}(\text { bin })}{\operatorname{rate}^{\mathrm{MC}}(\text { bin })}\right) \times \operatorname{Model}_{\mathrm{Rn}}(\text { bin })
$$

This uncertainty computation suffer from a slight bias as the model is applied on the true MC position, whereas the rate are calculated using reconstructed position. We believe this bias induces an over estimation of the uncertainty. Fig. 7 and 8 show the model uncertainty for each bin in 2D and 1D, respectively. The average uncertainty from the Rn model is estimated using the RMS of the 1D distribution as $\Delta \mathrm{C}_{\mathrm{Rn}}=0.1 \mathrm{mBq} / \mathrm{m}^{3}$. The main uncertainty on the modeled $\mathrm{Rn}$ concentration appears around $\mathrm{Z}=-10 \mathrm{~m}$ which correspond to the transition between the Rn-rich bottom of the detector and the Rn-less center of the detector.

\section{Discussion about the ${ }^{208}$ Tl decays}

In order to improve the Rn model and extend it on the full fiducial volume of the Super-K detector, an improved understanding of the ${ }^{208} \mathrm{Tl}$ BG is needed. Simulations of this BG have been 


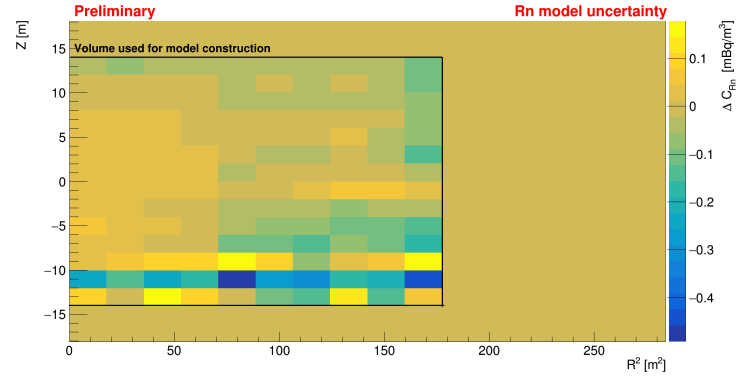

Figure 7: 2D map of the Rn model uncertainty, the region close to the border of the detector was excluded in order to reduce the impact of the ${ }^{208} \mathrm{Tl}$ decays.

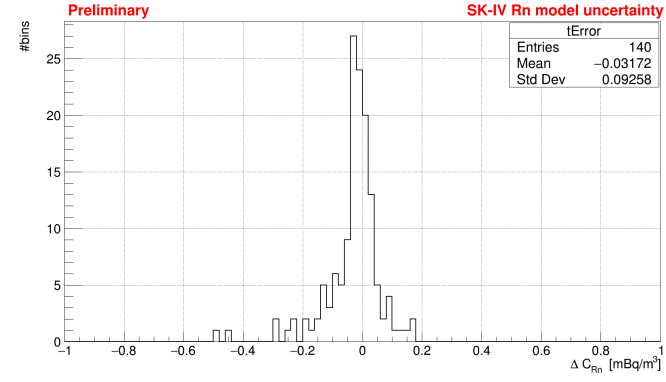

Figure 8: $1 \mathrm{D}$ projection of the 2D uncertainty map shown in Fig. 7

performed, but large statistics is need as only $10^{-6} \%$ of the simulated decay pass the selection criteria. Preliminary results indicate a relatively good agreement with data when added to the Rn model, as shown in Fig. 9 and 10. However, these studies also indicate the ${ }^{208} \mathrm{Tl}$ was overestimated at the top and bottom border of the detector, and underestimated above the convection front at $\mathrm{Z}=-10 \mathrm{~m}$.

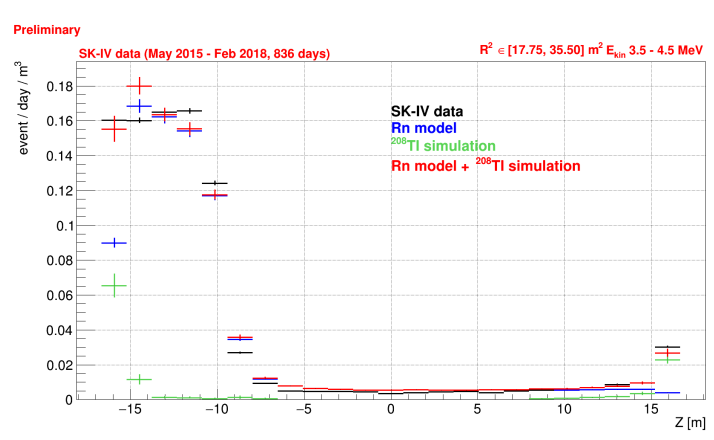

Figure 9: Event rate distribution in a Z-layer for SK-IV data (black), Rn model based MC (blue), and ${ }^{218} \mathrm{Tl}$ MC (green). The red distribution represent the sum of the Rn model based MC and of the ${ }^{218} \mathrm{Tl}$ MC.

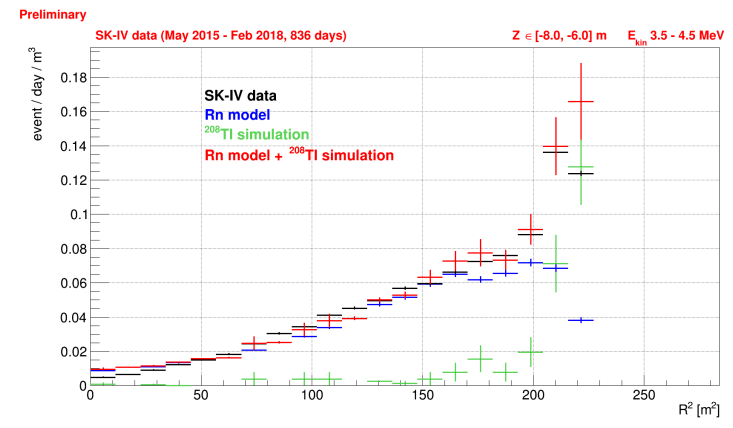

Figure 10: Event rate distribution in a $\mathrm{R}^{2}$-layer for SK-IV data (black), Rn model based MC (blue), and ${ }^{218} \mathrm{Tl}$ MC (green). The red distribution represent the sum of the Rn model based MC and of the ${ }^{218} \mathrm{Tl}$ MC.

\section{Conclusions and perspectives}

The Rn distribution model presented in this study is a first step allowing to improve our understanding of the Rn BG in the Super-K detector. As highlighted in Section 5, the ${ }^{208} \mathrm{Tl}$ was not properly estimated in some area of the detector. We are going to develop a new version of the Rn distribution model taking into account these new information, as well as increasing the statistics of our ${ }^{208} \mathrm{Tl}$ sample. 
In the future, this model of the Rn distribution could be used to develop new BG rejection method against Rn, as well as to estimate the impact of the Rn BG in future detector like HyperKamiokande [9].

\section{References}

[1] S. Fukuda et al. (the Super-Kamiokande collaboration), The Super-Kamiokande detector, Nucl. Instrum. Meth. A 501 (2003) 418-462

[2] Y. Nakano et al., Measurement of the radon concentration in purified water in the Super-Kamiokande IV detector, Nucl. Instrum. Meth. A 977, 164297 (2020) [physics.ins-det/1910.03823]

[3] Y. Takeuchi et al., Measurement of radon concentrations at Super-Kamiokande, Physics Letters B 452 (1999) 418-424 [hep-ex/9903006]

[4] K. Abe et al. (the Super-Kamiokande collaboration), Solar Neutrino Measurements in SuperKamiokande-IV, Phys.Rev.D 94 (2016) 5, 052010 [hep-ex/1606.07538]

[5] S.Agostinelli et al., Geant4-a simulation toolkit, Nucl. Instrum. Meth. A 506 (2003) 250-303

[6] J. Allison et al., Geant4 developments and applications, IEEE Transactions on Nuclear Science 53 (2006) 8999460

[7] J. Allison et al., Recent developments in Geant4, Nucl. Instrum. Meth. A 835 (2016) 186-225

[8] S. Syahrir, Transport of Radon in Still Water under Steady-State and Transient Conditions, Ph.D. thesis (2005) [ISBN：9780542053573]

[9] K. Abe et al. (the Hyper-Kamiokande proto-collaboration), Hyper-Kamiokande Design Report , [physics.ins-det/1805.04163] 


\section{Full Authors List: Super-Kamiokande Collaboration}

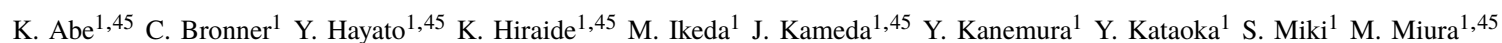

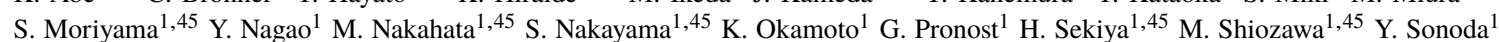
Y. Suzuki ${ }^{1}$ A. Takeda ${ }^{1,45}$ Y. Takemoto ${ }^{1}$ A. Takenaka ${ }^{1}$ H. Tanaka ${ }^{1}$ S. Watanabe ${ }^{1}$ T. Yano ${ }^{1}$ S. Han ${ }^{2}$ T. Kajita ${ }^{2,45}$ K. Okumura ${ }^{2,45}$ T. Tashiro ${ }^{2}$ J. Xia ${ }^{2}$ G. D. Megias ${ }^{3}$ L. Labarga ${ }^{4}$ Ll. Marti ${ }^{4}$ B. Zaldivar ${ }^{4}$ B. W. Pointon ${ }^{6,49}$ E. Kearns ${ }^{5,45}$ J. L. Raaf ${ }^{5}$ L. Wan ${ }^{5}$ T. Wester ${ }^{5}$

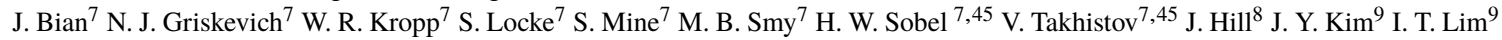
R. G. Park ${ }^{9}$ B. Bodur ${ }^{10}$ K. Scholberg ${ }^{10}$ C. W. Walter ${ }^{10,45}$ L. Bernard ${ }^{11}$ A. Coffani ${ }^{11}$ O. Drapier ${ }^{11}$ S. El Hedri ${ }^{11}$ A. Giampaolo ${ }^{11}$ M. Gonin ${ }^{11}$ Th. A. Mueller ${ }^{11}$ P. Paganini ${ }^{11}$ B. Quilain ${ }^{11}$ T. Ishizuka ${ }^{12}$ T. Nakamura ${ }^{13}$ J. S. Jang ${ }^{14}$ J. G. Learned ${ }^{15}$ S. Cao ${ }^{16}$ L. H. V. Anthony ${ }^{17}$ D. Martin ${ }^{17}$ M. Scott ${ }^{17}$ A. A. Sztuc ${ }^{17}$ Y. Uchida ${ }^{17}$ V. Berardi ${ }^{18}$ M. G. Catanesi ${ }^{18}$ E. Radicioni ${ }^{18}$ N. F. Calabria ${ }^{19}$ L. N. Machado ${ }^{19}$ G. De Rosa $^{19}$ G. Collazuol ${ }^{20}$ F. Iacob $^{20}$ M. Lamoureux ${ }^{20}$ M. Mattiazzi ${ }^{20}$ N. Ospina ${ }^{20}$ L. Ludovici ${ }^{21}$ Y. Maekawa $^{22}$ Y. Nishimura ${ }^{22}$ M. Friend ${ }^{23}$ T. Hasegawa ${ }^{23}$ T. Ishida ${ }^{23}$ T. Kobayashi ${ }^{23}$ M. Jakkapu ${ }^{23}$ T. Matsubara ${ }^{23}$ T. Nakadaira ${ }^{23}$ K. Nakamura ${ }^{23,45}$ Y. Oyama ${ }^{23}$ K. Sakashita ${ }^{23}$ T. Sekiguchi ${ }^{23}$ T. Tsukamoto ${ }^{23}$ T. Boschi ${ }^{24}$ F. Di Lodovico ${ }^{24}$ J. Gao $^{24}$ J. Migenda ${ }^{24}$ M. Taani ${ }^{24}$ S. Zsoldos ${ }^{24}$ Y. Nakano ${ }^{25}$ H. Ozaki ${ }^{25}$ A. T. Suzuki ${ }^{25}$ Y. Takeuchi ${ }^{25,45}$ S. Yamamoto ${ }^{25}$ Y. Kotsar ${ }^{25}$ A. Ali ${ }^{26}$ J. Feng $^{26}$ T. Kikawa ${ }^{26}$ M. Mori $^{26}$ T. Nakaya ${ }^{26,45}$ R. A. Wendell ${ }^{26,45}$ K. Yasutome ${ }^{26}$ P. Fernandez ${ }^{28}$ N. McCauley ${ }^{28}$ P. Mehta ${ }^{28}$ K. M. Tsui ${ }^{27}$ Y. Fukuda ${ }^{28}$ Y. Itow ${ }^{29,30}$ H. Menjo ${ }^{29}$ K. Sato ${ }^{29}$ J. Lagoda ${ }^{31}$ S. M. Lakshmi ${ }^{31}$ P. Mijakowski ${ }^{31}$ J. Zalipska ${ }^{31}$ J. Jiang ${ }^{32}$ C. K. Jung ${ }^{32}$ C. Vilela ${ }^{32}$ M. J. Wilking ${ }^{32}$ C. Yanagisawa ${ }^{32}$ M. Harada ${ }^{33}$ H. Ishino ${ }^{33}$ S. Ito ${ }^{33}$ H. Kitagawa ${ }^{33}$ Y. Koshio ${ }^{33,45}$ W. Ma ${ }^{33}$ N. Piplani ${ }^{33}$ S. Sakai ${ }^{33}$ G. Barr ${ }^{34}$ D. Barrow $^{34}$ L. Cook ${ }^{34,45}$ A. Goldsack ${ }^{34,45}$ S. Samani ${ }^{34}$ D. Wark ${ }^{34,39}$ F. Nova ${ }^{35}$ J. Y. Yang ${ }^{36}$ S. J. Jenkins ${ }^{37}$ M. Malek ${ }^{37}$ J. M. McElwee ${ }^{37}$ O. Stone ${ }^{37}$

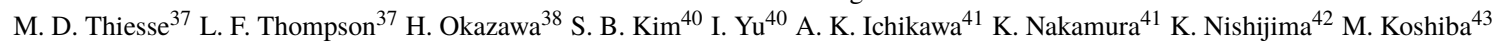
K. Iwamoto ${ }^{44}$ Y. Nakajima ${ }^{44}$ N. Ogawa ${ }^{44}$ M. Yokoyama ${ }^{44,45}$ K. Martens ${ }^{45}$ M. R. Vagins ${ }^{45,7}$ M. Kuze ${ }^{46}$ S. Izumiyama ${ }^{46}$ M. Inomoto $^{47}$ M. Ishitsuka ${ }^{47}$ H. Ito ${ }^{47}$ T. Kinoshita ${ }^{47}$ R. Matsumoto ${ }^{47}$ M. Shinoki ${ }^{47}$ T. Suganuma ${ }^{47}$ J. F. Martin ${ }^{48}$ H. A. Tanaka ${ }^{48}$ T. Towstego $^{48}$ R. Akutsu ${ }^{49}$ P. de Perio ${ }^{49}$ M. Hartz ${ }^{49}$ A. Konaka ${ }^{49}$ N. W. Prouse ${ }^{49}$ S. Chen ${ }^{50}$ B. D. Xu ${ }^{50}$ M. Posiadala-Zezula ${ }^{51}$ D. Hadley ${ }^{52}$ M. O'Flaherty ${ }^{52}$ B. Richards ${ }^{52}$ B. Jamieson ${ }^{53}$ J. Walker ${ }^{53}$ A. Minamino ${ }^{54}$ G. Pintaudi ${ }^{54}$ S. Sano ${ }^{54}$ R. Sasaki ${ }^{54}$

${ }^{1}$ Kamioka Observatory, Institute for Cosmic Ray Research, University of Tokyo, Kamioka, Gifu 506-1205, Japan.

${ }^{2}$ Research Center for Cosmic Neutrinos, Institute for Cosmic Ray Research, University of Tokyo, Kashiwa, Chiba 277-8582, Japan.

${ }^{3}$ Institute for Cosmic Ray Research, University of Tokyo, Kashiwa, Chiba 277-8582, Japan.
${ }^{4}$ Department of Theoretical Physics, University Autonoma Madrid, 28049 Madrid, Spain.

${ }^{5}$ Department of Physics, Boston University, Boston, MA 02215, USA.

6 Department of Physics, British Columbia Institute of Technology, Burnaby, BC, V5G 3H2, Canada.

${ }^{7}$ Department of Physics and Astronomy, University of California, Irvine, Irvine, CA 92697-4575, USA

${ }^{8}$ Department of Physics, California State University, Dominguez Hills, Carson, CA 90747, USA.

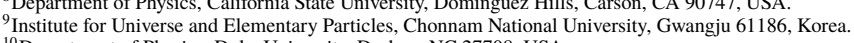

${ }^{10}$ Department of Physics, Duke University, Durham NC 27708, USA.

${ }^{11}$ Ecole Polytechnique, IN2P3-CNRS, Laboratoire Leprince-Ringuet, F-91120 Palaiseau, France.

${ }^{12}$ Junior College, Fukuoka Institute of Technology, Fukuoka, Fukuoka 811-0295, Japan.

${ }^{13}$ Department of Physics, Gifu University, Gifu, Gifu 501-1193, Japan.

${ }^{14}$ GIST College, Gwangju Institute of Science and Technology, Gwangju 500-712, Korea.

${ }^{15}$ Department of Physics and Astronomy, University of Hawaii, Honolulu, HI 96822, USA

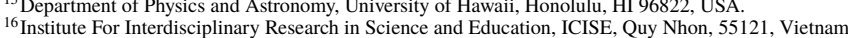

${ }^{17}$ Department of Physics, Imperial College London, London, SW7 2AZ, United Kingdom

${ }^{18}$ Dipartimento Interuniversitario di Fisica, INFN Sezione di Bari and Università e Politecnico di Bari, I-70125, Bari, Italy.

${ }^{19}$ Dipartimento di Fisica, INFN Sezione di Napoli and Università di Napoli, I-80126, Napoli, Italy.

${ }^{20}$ Dipartimento di Fisica, INFN Sezione di Padova and Università di Padova, I-35131, Padova, Italy.

${ }^{21}$ INFN Sezione di Roma and Università di Roma "La Sapienza", I-00185, Roma, Italy.

${ }^{22}$ Department of Physics, Keio University, Yokohama, Kanagawa, 223-8522, Japan.

${ }^{23}$ High Energy Accelerator Research Organization (KEK), Tsukuba, Ibaraki 305-0801, Japan

${ }^{24}$ Department of Physics, King's College London, London, WC2R 2LS, UK.

${ }^{25}$ Department of Physics, Kobe University, Kobe, Hyogo 657-8501, Japan.

${ }^{26}$ Department of Physics, Kyoto University, Kyoto, Kyoto 606-8502, Japan.

${ }^{27}$ Department of Physics, University of Liverpool, Liverpool, L69 7ZE, United Kingdom.

${ }^{28}$ Department of Physics, Miyagi University of Education, Sendai, Miyagi 980-0845, Japan.

${ }^{29}$ Institute for Space-Earth Environmental Research, Nagoya University, Nagoya, Aichi 464-8602, Japan.

${ }^{30}$ Kobayashi-Maskawa Institute for the Origin of Particles and the Universe, Nagoya University, Nagoya, Aichi 464-8602, Japan.

${ }^{31}$ National Centre For Nuclear Research, 02-093 Warsaw, Poland.

${ }^{32}$ Department of Physics and Astronomy, State University of New York at Stony Brook, NY 11794-3800, USA

${ }^{33}$ Department of Physics, Okayama University, Okayama, Okayama 700-8530, Japan

${ }^{34}$ Department of Physics, Oxford University, Oxford, OX1 3PU, United Kingdom.

${ }^{35}$ Rutherford Appleton Laboratory, Harwell, Oxford, OX11 0QX, UK.

${ }^{36}$ Department of Physics, Seoul National University, Seoul 151-742, Korea.

${ }^{37}$ Department of Physics and Astronomy, University of Sheffield, S3 7RH, Sheffield, United Kingdom.

${ }^{38}$ Department of Informatics in Social Welfare, Shizuoka University of Welfare, Yaizu, Shizuoka, 425-8611, Japan.

${ }^{39}$ Department of Informatics in Social Welfare, Shizuoka University of Welfare, Yaizu, Shizuoka, 425-8611, Japan.
${ }^{40}$, Rutherford Appleton Laboratory, Harwell Oxford, and Daresbury Laboratory, Warrington, OX11 0QX, United Kingdom.

${ }^{40}$ Department of Physics, Sungkyunkwan University, Suwon 440-746, Korea.

${ }^{41}$ Department of Physics, Faculty of Science, Tohoku University, Sendai, Miyagi, 980-8578, Japan.

${ }^{42}$ Department of Physics, Tokai University, Hiratsuka, Kanagawa 259-1292, Japan.

${ }^{43}$ The University of Tokyo, Bunkyo, Tokyo 113-0033, Japan.

${ }^{44}$ Department of Physics, University of Tokyo, Bunkyo, Tokyo 113-0033, Japan.

${ }^{45}$ Kavli Institute for the Physics and Mathematics of the Universe (WPI), The University of Tokyo Institutes for Advanced Study, University of Tokyo, Kashiwa, Chiba 277-8583, Japan.

${ }^{46}$ Department of Physics,Tokyo Institute of Technology, Meguro, Tokyo 152-8551, Japan.

${ }^{47}$ Department of Physics, Faculty of Science and Technology, Tokyo University of Science, Noda, Chiba 278-8510, Japan.

${ }^{48}$ Department of Physics, University of Toronto, ON, M5S 1A7, Canada.

${ }^{49}$ TRIUMF, 4004 Wesbrook Mall, Vancouver, BC, V6T2A3, Canada.

${ }^{50}$ Department of Engineering Physics, Tsinghua University, Beijing, 100084, China.

${ }^{51}$ Faculty of Physics, University of Warsaw, Warsaw, 02-093, Poland.

${ }^{52}$ Department of Physics, University of Warwick, Coventry, CV4 7AL, UK

${ }^{53}$ Department of Physics, University of Winnipeg, MB R3J 3L8, Canada.

${ }^{54}$ Department of Physics, Yokohama National University, Yokohama, Kanagawa, 240-8501, Japan.

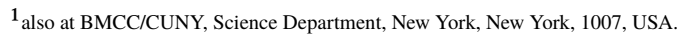

${ }^{2}$ Deceased. 